\title{
Sub-Nyquist sampling and detection in Costas coded pulse compression radars
}

Adnan Hanif ${ }^{*}$, Atif Bin Mansoor ${ }^{1}$ and Ali Shariq Imran ${ }^{2}$

\begin{abstract}
Modern pulse compression radar involves digital signal processing of high bandwidth pulses modulated with different coding schemes. One of the limiting factors in the radar's design to achieve desired target range and resolution is the need of high rate analog-to-digital $(A / D)$ conversion fulfilling the Nyquist sampling criteria. The high sampling rates necessitate huge storage capacity, more power consumption, and extra processing requirement. We introduce a new approach to sample wideband radar waveform modulated with Costas sequence at a sub-Nyquist rate based upon the concept of compressive sensing (CS). Sub-Nyquist measurements of Costas sequence waveform are performed in an analog-to-information (A/I) converter based upon random demodulation replacing traditional A/D converter. The novel work presents an 8-order Costas coded waveform with sub-Nyquist sampling and its reconstruction. The reconstructed waveform is compared with the conventionally sampled signal and depicts high-quality signal recovery from sub-Nyquist sampled signal. Furthermore, performance of CS-based detections after reconstruction are evaluated in terms of receiver operating characteristic (ROC) curves and compared with conventional Nyquist-rate matched filtering scheme.
\end{abstract}

Keywords: Compressive sensing, Costas waveform, Analog-to-information conversion, ROC curves

\section{Introduction}

Modern pulse compression radars transmit wideband pulse (linear chirp, binary phase codes, Costas codes, etc.), and perform compression of returned pulse digitally after high-rate analog-to-digital (A/D) conversion stage. The conventional A/D converters follow Nyquist sampling theorem, which requires uniform sampling rate at least twice of the signal bandwidth for guaranteed reconstruction of the band-limited signal [1]. Adequate A/ $\mathrm{D}$ conversion of wideband radar return signal requires both high sampling frequency and large dynamic range. In the radars employing digital pulse compression, one of the bottlenecks in achieving the desired range resolution is high-rate $\mathrm{A} / \mathrm{D}$ conversion, which in many cases is either beyond technological limits or is too expensive.

Recently, a leap in signal sensing realm is made by the promising approach of compressive sensing (CS), which is mainly supported by the positive theoretical and experimental results in [2-6]. It enables sampling at a rate comparable to signal's information rate. In CS, an

\footnotetext{
* Correspondence: adnanengineer@gmail.com

${ }^{1}$ Air University, Islamabad, Pakistan

Full list of author information is available at the end of the article
}

incoherent linear projection is employed to acquire an accurate representation of compressible signal directly using few measurements, much lesser than the number prescribed by the Nyquist theorem. The signal is then recovered from undersampled measurements by solving an inverse problem either through a linear program or a greedy pursuit [7].

Many recent techniques employ CS to sample sparse signal of interest at sub-Nyquist rate using a low-rate sampler as in analog-to-information (A/I) converter. Two popular A/I approaches are periodic nonuniform sampling [8] and random demodulator scheme [9] with its hardware implementation in [10]. Another A/I model, modulation wideband converter (MWC) [11] can undersample analog multiband signal along with spectrum-blind recovery. CS applications to radar are mainly focused to hard target radar [12] or multipleinput-multiple-output (MIMO) radar [13] exploiting sparsity in the target range, Doppler, or angle space. Similarly, CS-based radar imaging using synthetic aperture radar (SAR) for terrain mapping [14] and ultrawideband radar for through-the-wall target detection [15] are of major interests. Promising research, however, 


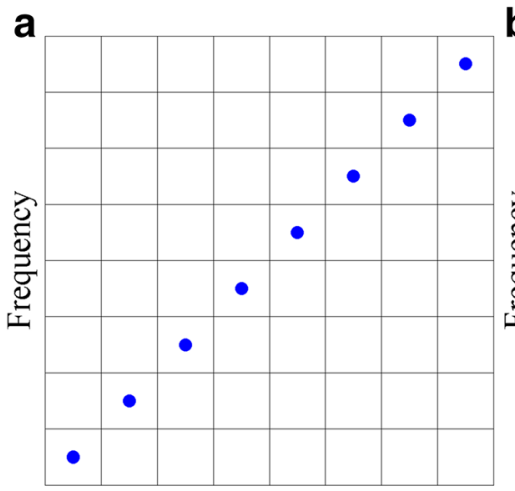

Time b

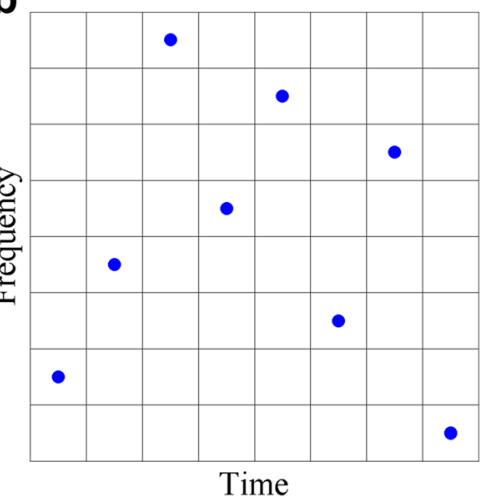

Fig. 1 Frequency assignment of eight sub-pulses. a Stepped linear-frequency modulation (SLFM) waveform. b 8-order Costas coded pulse

in employing CS to reduce radar receiver complexity and its A/D converter sampling rate [16] exploiting radar waveform characteristics are limited.

One approach in pulse compression radar waveform design that achieves high target range and Doppler resolution is the Costas sequence, introduced by J. Costas [17]. The basic building block of Costas waveform is a simple frequency-hopped signal of $N$ contiguous sub-pulses each having distinct frequencies, which are selected through a special class of $N \times N$ permutation matrices known as Costas arrays [18]. A class of Costas sequences for which corresponding ambiguity function approaches an ideal or a thumbtack-like response is called Costas codes [19]. For high-resolution delay-Doppler imaging radars, transmitting waveform of $N$ sub-pulses with proper frequency-shift sequence have Pulse Compression Ratio (PCR) of $N^{2}$ [20].

In this paper, we propose a novel approach utilizing the concept of compressive sensing to digital radar signal processing that employs Costas codes as the basis functions for pulse compression. We exploited the timefrequency sparsity of Costas sequence in transform domain (Gabor dictionary) to represent the received signal with fewer measurements than traditionally required. The fewer sampled data implies significant reduction in memory and power consumption. The results acquired from undersampling of radar returned waveform in A/I converter via random demodulation, and then its recovery by solving the convex optimization problem shows promising Costas sequence reconstructions. We have evaluated the proposed approach by comparing the CSbased signal recovery against conventional discrete sample reconstruction in terms of signal-to-noise ratio (SNR) and mean squared error (MSE). Finally, detection performance of CS-based Costas coded radar is analyzed through Monte Carlo simulations and is compared with conventional matched filtering (MF)-based radar detector by means of receiver operating characteristic (ROC) curves.

The paper is further organized as follows. In Section 2, we explain the generation of Costas waveform followed by a discussion on discrete-time CS theory. In Section 3, we look into sparsity in Costas waveform and present mathematical framework of A/I model for low-rate sampling of the continuous-time waveform and its subsequent reconstruction in transform basis. In Section 4, we lay ground work for ROC curves based performance analysis of CS-based detection scheme. Experimentation and results are discussed in Section 5. The paper is concluded in Section 6.

\section{Background}

\subsection{Costas sequence waveform}

The Costas sequence modulated waveform, transmitted by digital pulse compression radars, of pulse width $T$ seconds, and can be regarded as a frequency hopping signal of $N$ equal-length sub-pulses with $k$ th sub-pulse being frequency modulated with frequency $f_{k}$ with respect to carrier frequency $f_{0}$. In various time slots, frequencies to be placed are determined via sequence of ordered integers, $S(k), k=1, \ldots, N$. The sequence $S(k)$ for which the corresponding ambiguity function approaches an ideal or a "thumbtack" response is called Costas code.

The expression for the frequency $f_{k}$ is:

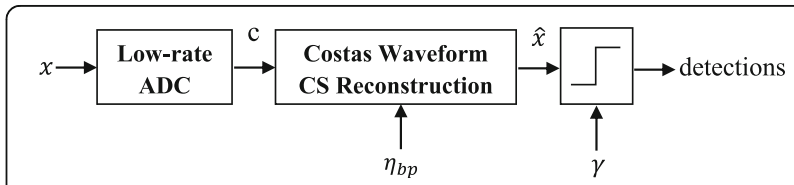

Fig. 2 Detection scheme involving separate detector after noisy CS reconstructions 

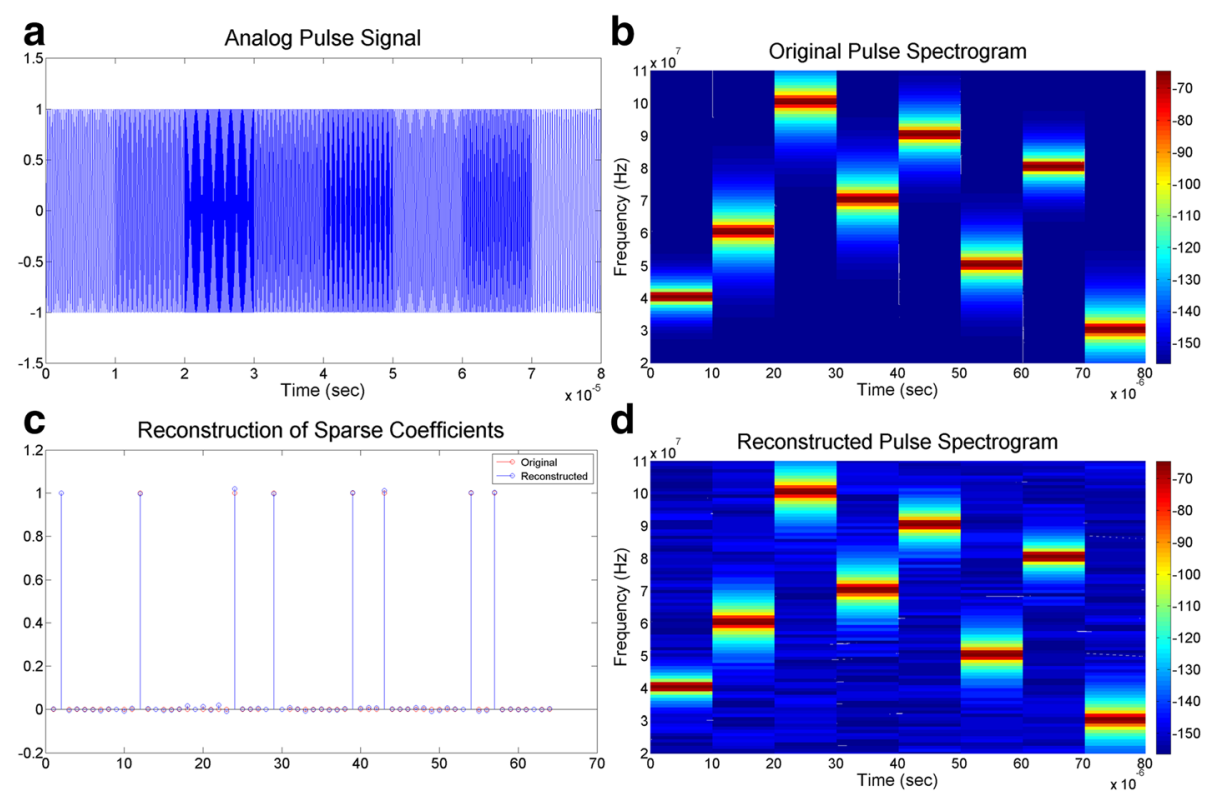

Fig. 3 8-order Costas coded pulse sampled at $4 \times$ sub-Nyquist rate: a Original wideband analog signal. b Original signal spectrogram. c Original and reconstructed sparse vectors. $\mathbf{d}$ Reconstructed signal spectrogram

$$
f_{k}=S(k) \frac{B}{N} \quad k=1, \ldots, N
$$

where $B$ is the approximate signal bandwidth. The Costas waveform can be written as:

$$
x(t)=\sum_{k=1}^{N} u\left(\frac{k T}{N}-t\right) \cos \left[\left(f_{0}+f_{k}\right) t+\theta_{k}\right]
$$

where

$$
u(t)=\left\{\begin{array}{cc}
1 & 0 \leq t \leq T / N \\
0 & \text { elsewhere }
\end{array}\right.
$$

and $f_{0}$ and $\theta_{k}$ are the carrier frequency and initial phase, respectively.

The Costas sequence can also be expressed in a convenient way through a $N \times N$ matrix shown in Fig. 1, where $N$ rows are used to denote the sub-pulses and the $N$ columns are used to denote the stepped frequency. A "dot" indicates the frequency value assigned to the associated sub-pulse. A near thumbtack response as obtained

Table 1 SNR (dB) and MSE for 8-sub-pulsed radar waveform

\begin{tabular}{lccccc}
\hline $\begin{array}{l}\text { Sub- } \\
\begin{array}{l}\text { Nyquist } \\
\text { rate }\end{array}\end{array}$ & SNR $(\mathrm{dB})$ & & & MSE & \\
& Costas coded & SLFM & & Costas coded & SLFM \\
\hline $2 \times$ & 39.4 & 38.6 & & $5.74 \mathrm{e}-5$ & $6.90 \mathrm{e}-5$ \\
$4 \times$ & 36.6 & 36.0 & & $1.09 \mathrm{e}-4$ & $1.25 \mathrm{e}-4$ \\
$8 \times$ & 33.3 & 32.9 & & $2.34 \mathrm{e}-4$ & $2.56 \mathrm{e}-4$ \\
$16 \times$ & 28.4 & 27.2 & & $7.23 \mathrm{e}-4$ & $9.53 \mathrm{e}-4$ \\
\hline
\end{tabular}

by Costas indicates the placement of one and only one frequency per time slot (row) and per frequency slot (column) [17]. Figure 1a shows the frequency assignment with a stepped linear-frequency modulation (SLFM) whereas in Fig. 1b, the frequency assignments are chosen in a random fashion, according to some predetermined rule, in 8-order Costas array.

According to the traditional Shannon/Nyquist theorem, the sampling of such waveform should be at least twice of its maximum bandwidth to avoid aliasing [1]. However, since the signal has distinct frequency components localized in time, we exploited sparse nature of Costas code by employing the concept of compressive sensing.

\subsection{Theory of compressive sensing}

Compressive sensing (CS) allows the acquisition of onedimensional discrete-time signal of length $N$ indexed as $x(n), n=1, \ldots, N$ which is compressible in transform basis $\Psi$ that provides a $K$-sparse representation of $x$ as:

$$
x=\sum_{n=1}^{N} b_{n} \psi_{n}=\sum_{i=1}^{K} b\left(n_{i}\right) \psi_{n_{i}}
$$

where $x$ is a linear combination of $K$ basis vectors chosen from $\psi_{n}, n_{i}$ are the indices of those vectors, and $b_{n}$ are the weighting coefficients. By stacking the basis vectors as columns into the $N \times N$ sparsity basis matrix $\Psi=\psi_{1} \ldots \psi_{N}$, its matrix notation can be 

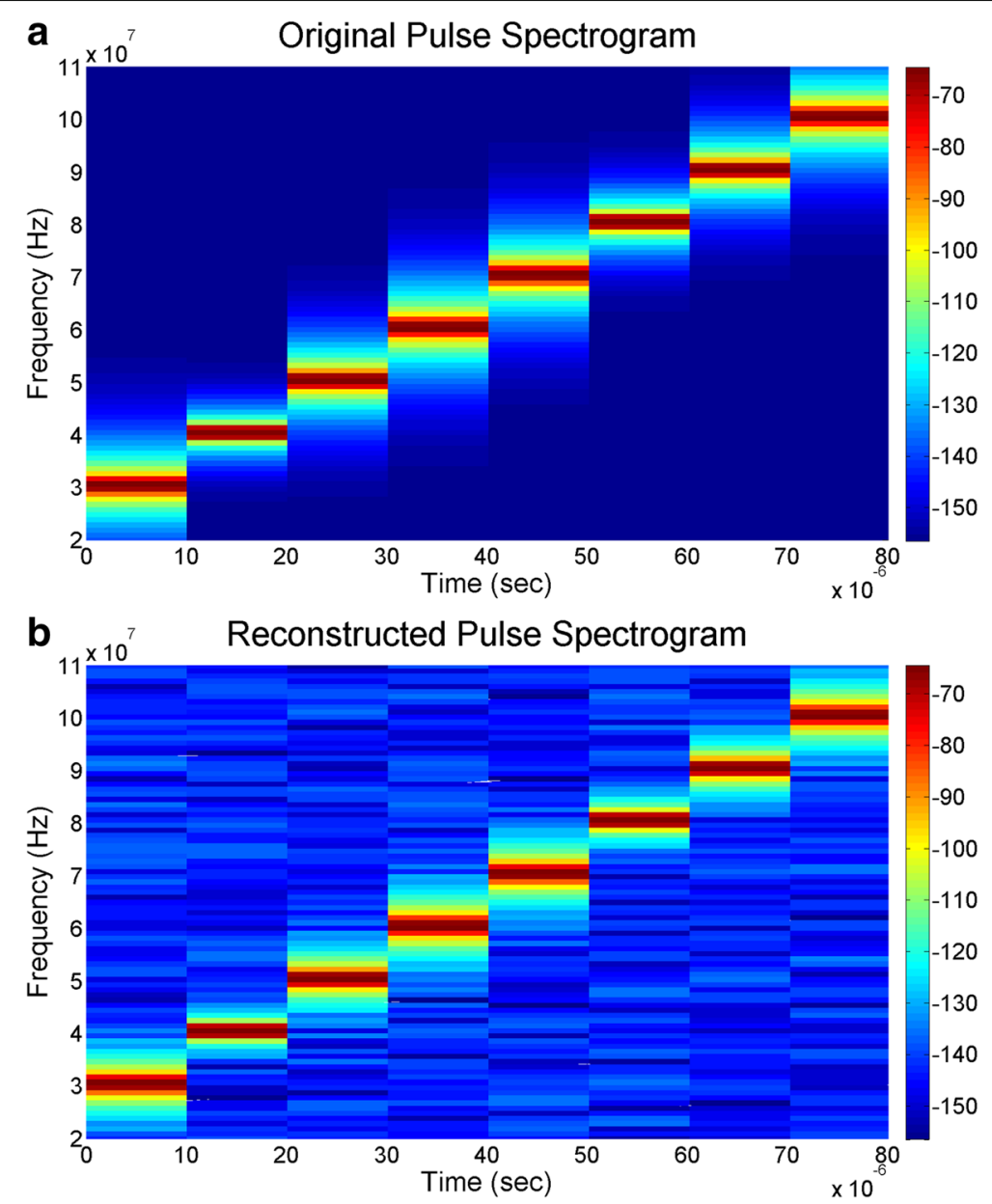

Fig. 4 SLFM waveform of eight sub-pulses sampled at $8 \times$ sub-Nyquist rate. a Spectrogram of original signal. b Spectrogram of A/l converted reconstructed signal

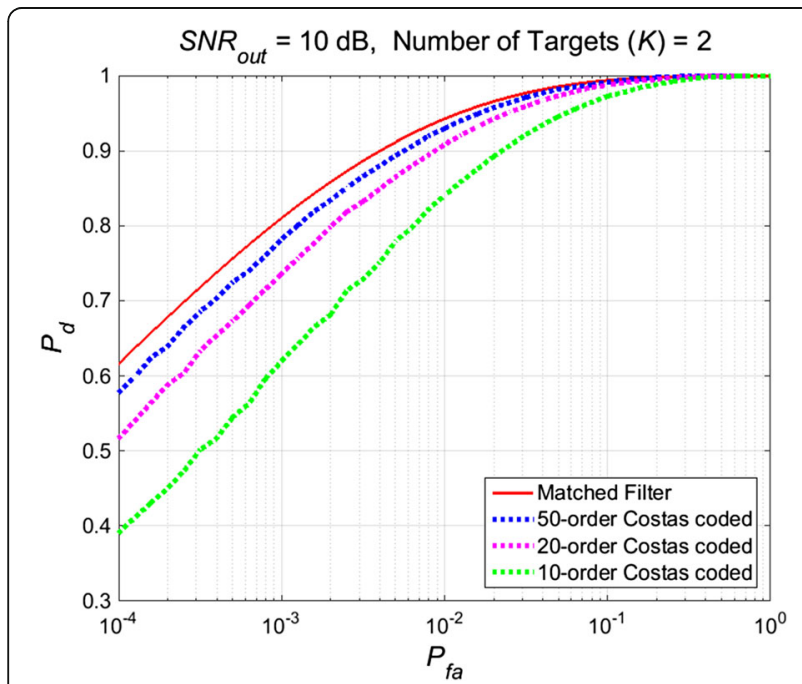

Fig. 5 Receiver operating characteristic (ROC) curves $\left(P_{\mathrm{d}}\right.$ vs $\left.P_{\mathrm{fa}}\right)$ at $\mathrm{SNR}=10 \mathrm{~dB}$ for varying order Costas coded pulses

$$
x=\Psi b
$$

where $b$ is an $N \times 1$ column vector consisting of terms with the $K$ largest magnitudes while setting all other terms to zero and $K \ll N$.

In compressive sensing, a signal that is compressible in one basis $\Psi$ can be recovered with $K$ largest $b_{n}$ 's from $M=O\left(K \log \frac{N}{K}\right)$ nonadaptive linear measurements on to a second basis $\Phi$ that is incoherent with the first basis $[2,21]$. By incoherent, it is meant that the rows of $\Phi$ do not provide a sparse representation of the columns of $\Psi$ and vice versa. Thus, instead of measuring the $N$-point signal $x$ directly, we take $M<N$ linear projections that can be expressed in matrix notation as:

$$
c=\Phi x=\Phi \Psi b=\mathbf{A} b
$$

where $c$ are the measurements in $M \times 1$ column vector, $\Phi$ is $M \times N$ measurement matrix, and $\mathbf{A}=\Phi \Psi$ we define is a $M \times N$ matrix which when holds the restricted isometry property (RIP) recovers the signal $x$ with high 


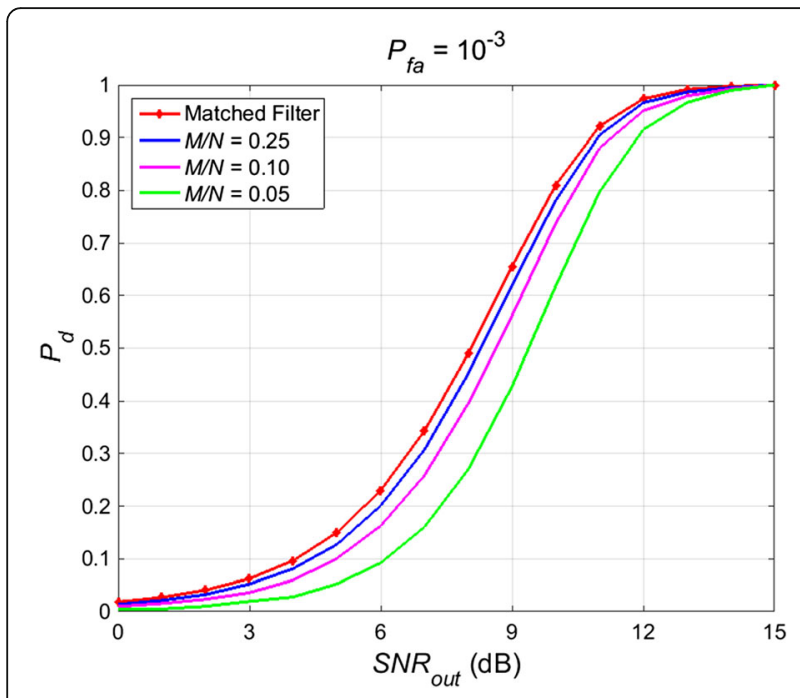

Fig. $6 P_{\mathrm{d}}$ VS SNR $\mathrm{R}_{\text {out }}$ performance at $P_{\mathrm{fa}}=10^{-3}$ with varying compressed sensing ratios $M / N$

quality from undersampled measurements $M[2,4,22]$. The RIP and incoherency are valid for many transform pairs such as sinusoids and wavelets and delta spikes and Fourier sinusoids. The recovery of the set of transform coefficients $b$ can be achieved through nonlinear optimization [23], by searching for the $b$ with the smallest $l_{1}$-norm that satisfies the $M$ observed measurements in $c$ :

$$
\hat{b}=\arg \min \|b\|_{1} \quad \text { such that } c=\mathbf{A} b
$$

This optimization problem, also known as Basis Pursuit [24], can be solved with conventional methods such as interior-point method. Alternatively, heuristic greedy algorithms such as least angle regression (LARS) [25] and orthogonal matching pursuit (OMP) [7] can also be applied at the expense of slightly more measurements.

\section{Signal sensing and recovery}

\subsection{Compressible Costas waveform}

The wideband Costas waveform in (1) possesses timefrequency sparsity in the sense that at each point in time, it is well approximated by few local sinusoids of constant frequency. The signals localized in the timefrequency domain have sparse representation under the Gabor transform, expressed as:

$$
\mathcal{V}_{g}(\tau, f)=\left\langle x(t), \psi_{\tau, f}(t)\right\rangle
$$

The coefficients of $\mathcal{V}_{g}$ are interpreted as the measure of the inner product of the signal with the Gabor atoms given by,

$$
\psi_{\tau, f}(t)=g(t-\tau) e^{ \pm j 2 \pi f t}
$$

while $g$ is Gabor window function with $\|g\|_{2}=1$ [26].

The wideband waveform when received by the radar receiver from a target possesses discrete, finite number of Gabor dictionary components, which when expressed in the form of (2), presents an ideal candidate for compressive sensing.

\subsection{Analog-to-information conversion}

Our proposed A/I-based radar signal acquisition system comprises of the proven steps of demodulation, filtering, and uniform sampling $[9,10]$. The signal is first modulated by a pseudo-random maximal-length sequence of \pm 1 's, alternating at or faster than the Nyquist frequency of the received signal, called the chipping sequence, $p(t)$. This demodulation spreads the frequency content of the signal to avoid aliasing in the second stage, which is a low-pass filter having an impulse response, $h(t)$. The signal is finally sampled at a lower rate $\mathscr{M}$ compared to Nyquist rate using a traditional low rate A/D converter.

For our system of sub-Nyquist sampling of a continuous-time Costas sequence waveform, the discrete measurement vector $c$ can be characterized as a linear transformation of the discrete coefficient vector $b$. In accordance with (3), this discrete transformation can be expressed as $M \times N$ reconstruction matrix A that combines two matrices: $\Psi$, which translates the discrete coefficient vector $b$ to an analog signal $x$, and $\Phi$, which translates the analog signal $x$ to the discrete set of measurements $c$.

The output $c[m]$, which is the result of convolution then demodulation and then sampling at rate $\mathscr{M}$ can be expressed as:

$$
c[m]=\int_{-\infty}^{\infty} x(\tau) p(\tau) h(t-\tau) d \tau
$$

such that $t=m \mathscr{M}$. As the Costas waveform expressed in (2) has discrete and sparse number of coefficients $\Psi$, therefore, (5) can be expanded and rearranged as:

$$
c[m]=\sum_{n=1}^{N} b_{n} \int_{-\infty}^{\infty} \psi_{n}(\tau) p(\tau) h(m \mathscr{M}-\tau) d \tau
$$

The reconstruction matrix A can be acquired from (6) by separating expression for each element $\alpha_{m, n} \in \mathbf{A}$ for row $m$ and column $n$ :

$$
\alpha_{m, n}=\int_{-\infty}^{\infty} \psi_{n}(\tau) p(\tau) h(m \mathscr{M}-\tau) d \tau
$$

The reconstruction of the Costas waveform is then performed by exploiting the dictionary of Gabor atoms and recovering the set of transform coefficients $b$ using nonlinear optimization algorithm involving least $l_{1}$-norm 
solution as in (4) to recover the signal directly in the sparse domain.

\section{CS-based detection}

In this section, we discuss updated CS model which we use to compare performance of CS-based Costas coded radar detector with that of matched filtering (MF) in classical radars. Since classical radar detection theory has since long been well established, detection properties of CS-based techniques are not yet very well known [27]. As CS approach involves new set of parameters which do not appear in conventional radar systems such as target sparseness $(K)$ and compression ratio $(M / N)$, our aim is to assess the effects of these parameters on the detection performance of CS-based Costas coded radar. To update the CS model for evaluating detector performance, we incorporate reconstruction threshold $\left(\eta_{b p}\right)$ in (4) so that recovery is performed as:

$$
\hat{b}=\arg \min \|b\|_{1} \quad \text { such that }\|c-\mathbf{A} b\|_{2} \leq \eta_{b p}
$$

where threshold $\eta_{b p}$ is proportional to noise standard deviation. This nonlinear optimization problem, as already mentioned, is known as Basis Pursuit (BP). In order to achieve desired $\left(P_{\mathrm{d}}, P_{\mathrm{fa}}\right)$ with an assigned SNR against selected number of measurements $M$ and threshold $\eta_{b p}$, we add a second detector after CS reconstruction as shown in Fig. 2. A separate detector after CS (tuning $\gamma$ in Fig. 2 reduces $P_{\mathrm{fa}}$ while maintaining high $P_{\mathrm{d}}$ gives better performance than using single detector alone [28].

We present a case of one-dimensional radar which is to determine the presence or absence of target in a given range bin. Consider an unambiguous mapping of target ranges to phases over the whole transmitted bandwidth requires $\mathrm{N}$-order Costas coded waveform, of duration $T$, at Nyquist rate. In a compressive sensing (CS) framework, we exploit the sparsity in target presence at a given range bin and reduce the number of discrete frequency steps in a transmitted Costas coded waveform to $M$. The frequencies in $M$-order Costas coded waveform are selected uniformly at random (with none repeated) out of $N$ frequencies with $M \ll N$, such that first and last frequencies in bandwidth are selected and that at least two of the adjacent transmitted frequencies have separation $\Delta f$ to ensure unambiguous range $\Delta R$ preserved. Moreover, to achieve a fair comparison with conventional detection, it is assumed that same total power is transmitted irrespective of the order of Costas code, i.e., transmitted pulse duration $T$ is to remain constant, having $M / T$ bandwidth of each sub-pulse in Costas waveform.
The compressed received signal of $M$-order Costas code is corrupted by additive Gaussian noise of variance $\sigma^{2}$. The $M \times N$ measurement matrix $\Phi$ is given as:

$$
\Phi=\frac{1}{\sqrt{M}}\left[\begin{array}{ccc}
e^{-j r_{1} k_{1} / N} & \ldots & e^{-j r_{N} k_{1} / N} \\
\vdots & \ddots & \vdots \\
e^{-j r_{1} k_{M} / N} & \cdots & e^{-j r_{N} k_{M} / N}
\end{array}\right]
$$

where $k_{m}=k_{0}+m 2 \pi / \Delta R$, here $m=1, \ldots, M$ is the wave number with $\Delta R=r_{N}-r_{0}$ and $r_{n}=r_{0}+n \delta R$, here $n=1$, ..., $N$ is the range bin index with $\delta R=\Delta R / N$.

Since the signal $x$ of length $N$ range bins is a sparse vector comprising of $K$ complex target amplitudes $a_{k}$ where $k=1, \ldots, K$ are indices corresponding to range bins where targets are located and zero elsewhere. Thus, $x$ $=\left[a_{1}, 0, a_{2}, 0, \ldots 0, a_{K}\right]$ is sparse directly in range domain which leads to identity matrix $I_{N}$ as basis matrix $\Psi$ and is therefore maximally incoherent with $\Phi$. The output signal-to-noise ratio $\left(\mathrm{SNR}_{\text {out }}\right)$, for each target, both for conventional and CS-based approach is given by:

$$
\mathrm{SNR}_{\text {out }}=\left|a_{k}\right|^{2} / \sigma^{2}
$$

where $\sigma^{2}$ is noise variance per sample. As output SNR is input to the detector and is independent of $M$ or $N$, it makes receiver operating characteristic (ROC)-based comparison more elegant, which is presented in next section.

\section{Experimental results and discussion}

\subsection{Costas waveform reconstruction performance}

We demonstrate the effectiveness of our approach by first compressive sensing a Costas coded radar waveform, taking its undersampled measurements in A/I converter. The pulsed signal recovery is then performed by solving convex optimization problem using CVX 2.1 package in MATLAB [29].

Firstly, 8-order Costas coded transmit pulse of radar operating in L-band having random stepped-frequency (Costas coded) modulation bandwidth of $80 \mathrm{MHz}$ with step of $10 \mathrm{MHz}$ is considered. The random hopping pattern satisfying the Costas conditions of having an ideal/ thumbtack-like ambiguity function response is used. The received pulsed echo after undergoing band-pass filtering and IF mixing is shown in analog form centered at $65 \mathrm{MHz}$ in Fig. 3a, and its corresponding spectrogram is depicted in Fig. 3b. The signal is then modulated with the pseudo-random chipping sequence of \pm 1's alternating at clock frequency of $1 \mathrm{GHz}$ in analog-toinformation (A/I) converter. The output of the demodulator is passed through a low-pass filter. Finally, the output is sampled in the presence of additive white Gaussian noise (AWGN) using a low-rate A/D converter at $40 \mathrm{M}$ samples/s, i.e., at $1 / 4$ times less than the 
traditional Nyquist sampling rate. The reconstruction of signal is then performed by exploiting the dictionary of Gabor atom frequencies, having potential to sparsely represent the signal of interest. By solving the $l_{1}$ - norm minimization problem in $\mathrm{cvx}$ using reconstruction matrix A, the signal is recovered directly in timefrequency domain. Figure 3c shows accurate recovery of sparse coefficients in dictionary of Gabor atom frequencies. The spectrogram, computed by squaring the magnitude of reconstruction, is shown in Fig. 3d. The reconstructed pulse spectrogram in Fig. 3d clearly displays high fidelity signal recovery when compared with original signal spectrogram shown in Fig. 3b.

To quantify the performance of Costas sequence sampled at sub-Nyquist rate, recovered signal is compared with conventionally sampled signal and, its signal-tonoise ratio (SNR) and mean squared error (MSE) is computed. The second and fourth column in Table 1 give the values of SNR and MSE, respectively, for $2 \times$, $4 \times, 8 \times$, and $16 \times$ sub-Nyquist rate sampling, which depicts high-quality signal restoration even for minimum sampled data.

In our second example, we performed compressive sensing of a stepped linear-frequency modulated (SLFM) pulse in the same L-band. The frequency modulates linearly, among eight steps, between the frequency limits as mentioned in our first experiment. Again, $2 \times, 4 \times, 8 \times$, and $16 \times$ sub-Nyquist rate sampling is performed in an $\mathrm{A} / \mathrm{I}$ converter. The reconstruction is performed by exploiting the same Gabor dictionary of atom frequencies using nonlinear recovery algorithm. The spectrogram of the original SLFM pulse and recovered timefrequency sequence from $8 \times$ sub-Nyquist rate samples is shown in Fig. 4.

The SNR and MSE performance as presented in column 3 and column 5, respectively, of Table 1 show highquality reconstructed waveform at different undersampling rates using $\mathrm{A} / \mathrm{I}$ converter. With decrease in the number of measurements acquired, the SNR of reconstructed signal slightly degrades as expected, but the reconstruction performance is still appreciable even for higher undersampling rates.

\subsection{ROC-based detection performance}

To better appreciate the performance of CS-based Costas coded radar, ROC curves for its detector are simulated and are compared with theoretical detection performance of matched filtering (MF) in classical radars under varying $M$ for a given SNR and $P_{\text {fa. }}$. Ten thousand Monte Carlo simulations are performed, and results are compared with an equal MF output SNR. The case is simulated for 2-point targets $(K=2)$ of constant amplitude, under ideal conditions that no sources of error other than noise are present. $N=200$ is used with varying $M / N$ ratio of $0.25,0.1$, and 0.05 , corresponding to 50-order, 20-order, and 10-order Costas coded waveforms respectively, of same pulse duration. The probability of detection $P_{\mathrm{d}}$ and probability of false alarm $P_{\mathrm{fa}}$ are calculated by counting the number of instances when targets-present return and targets-absent return exceed detector threshold $\gamma$, respectively, and normalizing them over the length of respective simulations round. $P_{\mathrm{d}}$ in ROC curves are then simulated by varying $P_{\mathrm{fa}}$ which is a function of threshold $\gamma$ over fixed SNR simulations output, or by varying SNR over fixed $P_{\mathrm{fa}}$ simulations output. Figure 5 shows the comparison of such simulated ROC curves with that of MF performance at a given $\mathrm{SNR}_{\text {out }}$ of about $10 \mathrm{~dB}$. Similarly, Fig. 6 depicts the performance of CS-based detection where $P_{\mathrm{d}}$ is plotted versus SNR for a fixed $P_{\mathrm{fa}}=10^{-3}$ for all values of $M / N$ ratio.

The above results clearly show that CS-based Costas coded radar performs very close to conventional optimum MF system. Moreover, ROC curves show graceful degradation of target detection probability as the number of measurements is decreased in comparison to what is required at Nyquist rate.

\section{Conclusions}

In this paper, we presented a novel application of compressive sensing in Costas coded pulse compression radars. The wideband radar return is sampled below Nyquist rate in real time using an analog-to-information (A/I) converter. The recovery in time-frequency domain is achieved by employing nonlinear recovery algorithm. The reconstruction of Costas waveforms with different sub-Nyquist sampling rates are demonstrated which results in significant reduction of A/D conversion bandwidth, memory capacity, and power consumption. The recovered signal holds high fidelity discrete frequency coding (Costas sequence) for pulse compression. The approach relaxes the bounds of using high-rate A/D converters for high bandwidth signal processing, in radars and other applications, having low "information rate." Moreover, a separate detector is used after CS reconstructions, and its performance is evaluated in terms of ROC curves through simulations. The analysis shows that even less order Costas coded waveforms in pulse compression radars depicts remarkable detection performance, quite comparable to that of conventional Nyquist-rate matched filtering.

\footnotetext{
Abbreviations

A/D: Analog-to-digital; A/l: Analog-to-information; AWGN: Additive white Gaussian noise; BP: Basis pursuit; CS: Compressive sensing; LARS: Least angle regression; MF: Matched filtering; MIMO: Multiple-input-multiple-output; MSE: Mean squared error; MWC: Modulation wideband converter; OMP: Orthogonal matching pursuit; PCR: Pulse compression ratio; RIP: Restricted isometry property; ROC: Receiver operating characteristic; SAR: Synthetic aperture radar; SLFM: Stepped linear-frequency modulation; SNR: Signal-to-noise ratio
} 


\section{Authors' contributions}

All authors read and approved the final manuscript.

\section{Competing interests}

The authors declare that they have no competing interests.

\section{Author details}

${ }^{1}$ Air University, Islamabad, Pakistan. ${ }^{2}$ Norwegian University of Science and Technology, Gjøvik, Norway.

Received: 9 June 2016 Accepted: 15 November 2016

Published online: 24 November 2016

\section{References}

1. CE Shannon, Communication in the presence of noise. Proc. IRE 37(1), 10-21 (1949)

2. DL Donoho, Compressed sensing. IEEE Trans. Inf. Theory 52(4), 1289-1306 (2006)

3. E Candès, J Romberg, Practical signal recovery from random projections. Proc SPIE Conf. Wavelet XI, 2005, p. 5914

4. E Candès, J Romberg, T Tao, Robust uncertainty principles: exact signal reconstruction from highly incomplete frequency information. IEEE Trans. Inf. Theory 52(2), 489-509 (2006)

5. E Candès, MB Wakin, An introduction to compressive sampling. IEEE Signal Process. Mag. 25(2), 21-30 (2008)

6. R Gribonval, M Nielsen, Sparse representations in unions of bases. IEEE Trans. Inf. Theory 49(12), 3320-3325 (2003)

7. JA Tropp, AC Gilbert, Signal recovery from partial information via orthogonal matching pursuit. IEEE Trans. Inf. Theory, 2005

8. JN Laska et al., Random sampling for analog-to-information conversion of wideband signals. IEEE Dallas CAS Workshop, 2006, pp. 119-122

9. S Kirolos et al., Analog-to-information conversion via random demodulation. IEEE Dallas CAS Workshop, 2006, pp. 71-74

10. JN Jason et al., Theory and implementation of an analog-to-information converter using random demodulation. IEEE Int. Symp. Circuits and Systems, 2007, pp. 1959-1962

11. M Mishali, YC Eldar, From theory to practice: sub-Nyquist sampling of sparse wideband analog signals. IEEE Journal of Selected Topics in Signal Process. 4(2), 375-391 (2010)

12. $\mathrm{H}$ Ender, On compressive sensing applied to radar. Signal Process. 90(5), 1402-1414 (2010)

13. Y Yu, AP Petropulu, HV Poor, CSSF MIMO RADAR: compressive-sensing and step-frequency based MIMO radar. IEEE Trans. Aerospace and Electronic Systems 48(2), 1490-1504 (2012)

14. $X$ Dong, $Y$ Zhang, A novel compressive sensing algorithm for SAR imaging. IEEE Journal of Selected Topics in Applied Earth Observations and Remote Sensing 7(2), 708-720 (2014)

15. B Zhang, W Wang, Through-wall detection of human being with compressed UWB radar data. EURASIP J. Wirel. Commun. Netw. 1, 162 (2013)

16. R Baraniuk, P Steeghs, Compressive radar imaging. IEEE Radar Conference, 2007, pp. 128-133, doi:10.1109/RADAR.2007.374203

17. JP Costas, A study of a class of detection waveforms having nearly ideal range-Doppler ambiquity properties. Proc. IEEE 72(8), 996-1009 (1984)

18. SW Golomb, T Herbert, Constructions and properties of Costas array. Proc. IEEE 72(9), 1143-1163 (1984)

19. BR Mahafza, Radar systems analysis and design using MATLAB. CRC Press, 2002, pp. 267-270, ISBN: 9781439884959

20. AW Rehaczek, Radar waveform selection - a simplified approach. IEEE Trans. Aerospace and Electronic Systems AES-7(6), 1078-1086 (1971)

21. E Candès, J Romberg, Quantitative robust uncertainty principles and optimally sparse decompositions. Found. Comp. Math. 6(2), 227-254 (2006)

22. R Baraniuk, M Davenport, R DeVore, M Wakin, A simple proof of the restricted isometry property for random matrices. Constr. Approx. 28(3), 253-263 (2008)

23. E Candès, J Romberg, $T$ Tao, Stable signal recovery from incomplete and inaccurate measurements. Commun. Pure Appl. Math. 59(8), 1207-1223 (2006)

24. S Chen, D Donoho, M Saunders, Atomic decomposition by basis pursuit SIAM J. Sci. Comput. 20(1), 33-61 (1999)

25. B Efron, T Hastie, I Johnstone, R Tibshirani, Least angle regression. Ann. Stat. 32, 407-499 (2004)
26. S Mallat, A wavelet tour of signal processing, 2nd edn. (Academic, San Diego, 1999)

27. L Anitori, M Otten, P Hoogeboom, Detection performance of Compressive Sensing applied to radar. IEEE Radar Conference, 2011, pp. 200-205, doi:10.1109/RADAR.2011.5960528

28. L Anitori, M Otten, W van Rossum, A Maleki, R Baraniuk, Compressive CFAR radar detection. IEEE Radar Conference, 2012, pp. 320-325, doi:10.1109/ RADAR.2012.6212158

29. CVX Research, Inc. CVX: Matlab software for disciplined convex programming, version 2.1, http://cvxr.com/cvx, Oct. 2014

\section{Submit your manuscript to a SpringerOpen ${ }^{\circ}$ journal and benefit from:}

- Convenient online submission

- Rigorous peer review

- Immediate publication on acceptance

- Open access: articles freely available online

- High visibility within the field

- Retaining the copyright to your article

Submit your next manuscript at $>$ springeropen.com 